\title{
Analysis of Deep Brain Stimulation Lead Targeting in the Stimulation of Anterior Nucleus of the Thalamus for Epilepsy Clinical Trial.
}

\author{
Robert E. Gross \\ Emory University \\ Robert S. Fisher \\ Stanford University \\ Michael R. Sperling \\ Thomas Jefferson University \\ Jonathon E. Giftakis \\ Aofdtwothifs Lhfed additional works at: https://jdc.jefferson.edu/neurologyfp

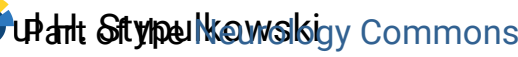 \\ Medtranis "know how access to this document benefits you
}

\section{Recommended Citation}

Gross, Robert E.; Fisher, Robert S.; Sperling, Michael R.; Giftakis, Jonathon E.; and Stypulkowski, Paul H., "Analysis of Deep Brain Stimulation Lead Targeting in the Stimulation of Anterior Nucleus of the Thalamus for Epilepsy Clinical Trial." (2021). Department of Neurology Faculty

Papers. Paper 257.

https://jdc.jefferson.edu/neurologyfp/257

This Article is brought to you for free and open access by the Jefferson Digital Commons. The Jefferson Digital Commons is a service of Thomas Jefferson University's Center for Teaching and Learning (CTL). The Commons is a showcase for Jefferson books and journals, peer-reviewed scholarly publications, unique historical collections from the University archives, and teaching tools. The Jefferson Digital Commons allows researchers and interested readers anywhere in the world to learn about and keep up to date with Jefferson scholarship. This article has been accepted for inclusion in Department of Neurology Faculty Papers by an authorized administrator of the Jefferson Digital Commons. For more information, please contact: JeffersonDigitalCommons@jefferson.edu. 


\section{Analysis of Deep Brain Stimulation Lead Targeting in the Stimulation of Anterior Nucleus of the Thalamus for Epilepsy Clinical Trial}

\section{Robert E. Gross, MD, PhD* \\ Robert S. Fisher, MD, PhD Michael R. Sperling, MD $\$$ Jonathon E. Giftakis, PhD (1)? Paul H. Stypulkowski, PhD" on behalf of the SANTÉ Study Group}

*Departments of Neurosurgery and $\mathrm{Ne}$ urology, Emory University, Atlanta, Georgia, USA; ${ }^{\ddagger}$ Department of Neurology and Neurological Sciences and by courtesy, Neurosurgery, Stanford, California, USA ${ }^{\S}$ Department of Neurology, Thomas Jeff erson University, Philadelphia, Pennsylvania, USA; " Medtronic, Inc., Minneapolis, Minnesota, USA

\section{Correspondence:}

Robert E. Gross, MD, PhD,

Emory University,

101 Woodruff Circle,

WMB 6311,

Atlanta, GA 30322, USA.

Email: rgross@emory.edu

Received, August 4, 2020

Accepted, January 24, 2021.

Published Online, June 23, 2021

(C) Congress of Neurological Surgeons 2021

This is an Open Access article distributed under the terms of the Creative

Commons Attribution-NonCommercial License (http://creativecommons. org/licenses/by-nc/4.0/), which permits non-commercial re-use, distribution, and reproduction in any medium, provided the original work is properly cited. For commercial re-use, please contact journals.permissions@oup.com

BACKGROUND: Deep brain stimulation (DBS) of the anterior nucleus of the thalamus (ANT) is an effective therapy for patients with drug-resistant focal epilepsy. Best practices for surgical targeting of the ANT can be refined as new information becomes available regarding effective stimulation sites.

OBJECTIVE: To conduct a retrospective analysis of the relationship between outcomes (seizure reduction during year 1 ) and DBS lead locations in subjects from the SANTÉ pivotal trial (Stimulation of ANT for Epilepsy) based upon recent clinical findings.

METHODS: Postoperative images from SANTÉ subjects $(n=101)$ were evaluated with respect to lead trajectory relative to defined anatomic landmarks. A qualitative scoring system was used to rate each lead placement for proximity to an identified target region above the junction of the mammillothalamic tract with the ANT. Each subject was assigned a bilateral lead placement score, and these scores were then compared to clinical outcomes.

RESULTS: Approximately $70 \%$ of subjects had "good" bilateral lead placements based upon location with respect to the defined target. These subjects had a much higher probability of being a clinical responder ( $>50 \%$ seizure reduction) than those with scores reflecting suboptimal lead placements $(43.5 \%$ vs $21.9 \%, P<.05)$.

CONCLUSION: Consistent with experience from more established DBS indications, our findings and other recent reports suggest that there may be specific sites within the ANT that are associated with superior clinical outcomes. It will be important to continue to evaluate these relationships and the evolution of other clinical practices (eg, programming) to further optimize this therapy.

KEY WORDS: Anterior nucleus of the thalamus, Deep brain stimulation, Epilepsy, Targeting

Neurosurgery 89:406-412, 2021

https://doi.org/10.1093/neuros/nyab186

www.neurosurgery-online.com

eep brain stimulation (DBS) of the anterior nucleus of the thalamus (ANT) was recently approved in the United States for partial onset epilepsy. The clinical trial (Stimulation of ANT for Epilepsy, SANTÉ) supporting this therapy was initiated in 2003 with results first published in 2010. ${ }^{1}$ As part of the original

ABBREVIATIONS: ANT, anterior nucleus of the thalamus; AP, anterior-posterior; DV, dorso-ventral; MCP, mid-commissural point; MTT, mammillothalamic tract; SANTÉ, Stimulation of ANT for Epilepsy; STN, subthalamic nucleus; VTA, volume of tissue activated

Neurosurgery Speaks! Audio abstracts available for this article at www.neurosurgery-online.com. analyses of the trial data, a detailed mapping of the active DBS contact locations was completed for each subject, using the standard anterior commissure-posterior commissure (AC-PC) based reference space, but comparison of active contact positions and clinical outcomes revealed no apparent relationship. Recently, several reports of ANT-DBS case series using advanced imaging have described considerable interpatient variability in the location of this nucleus in relationship to AC-PC reference space..$^{2-4}$ In addition to the interpatient variability in position of the ANT, they also noted considerable deviation compared to the Schaltenbrand-Wahren atlas, with the nucleus located more superior and anterior in most subjects as compared to the atlas specimens. 


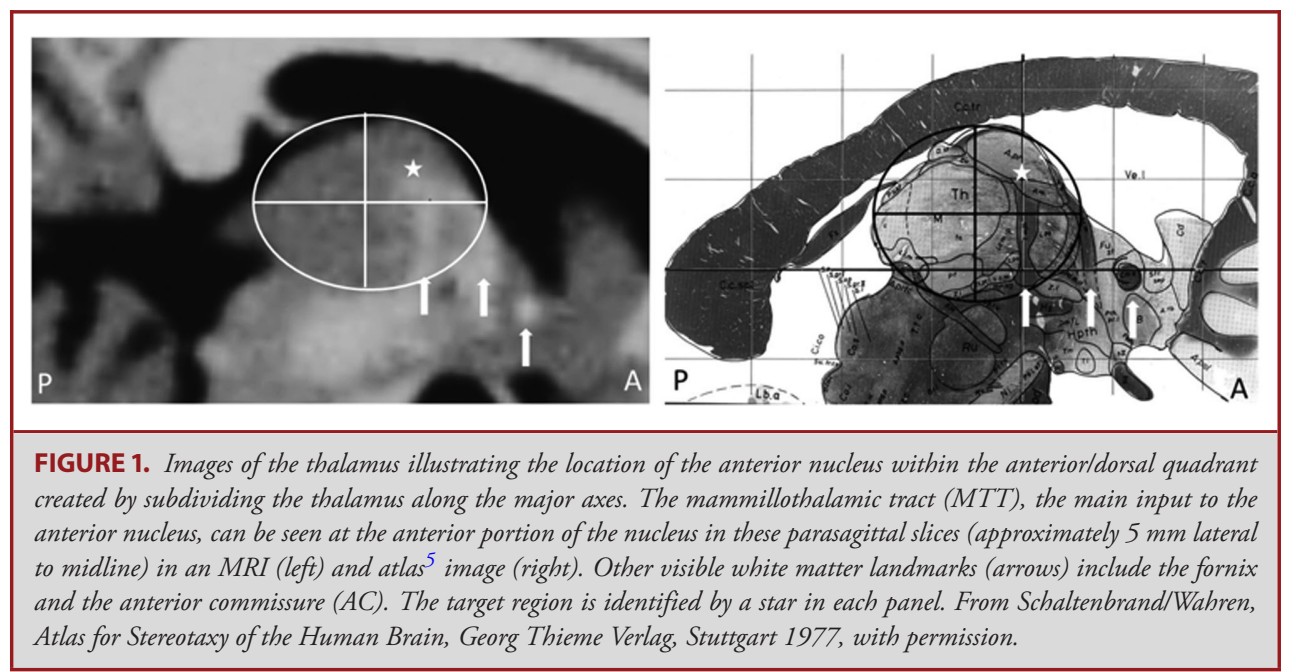

This was notable given that, in the SANTÉ trial, indirect targeting based upon coordinates derived from the Schaltenbrand-Wahren atlas was used to guide the initial coordinates for lead placement. Finally, these groups also identified a subregion target within the ANT, superior to the mammillothalamic tract (MTT) termination, that appeared to be associated with improved outcomes. These findings prompted us to perform a post hoc re-evaluation of the lead placements to determine whether there might be a relationship between patient-specific lead locations and clinical outcomes in the SANTÉ cohort.

\section{METHODS}

The SANTÉ trial (clinicaltrials.gov, identifier NCT00101933) was approved by the institutional review board (IRB) at all study centers, and subjects provided written informed consent before participation. Per the SANTÉ protocol, all subjects $(\mathrm{n}=110)$ had postoperative imaging and confirmation of bilateral DBS lead placement in the ANT before entering the randomized treatment phase. For the original assessment of lead positions, these image sets were used to determine the individual contact locations of the DBS leads (Model 3387, Medtronic) relative to the mid-commissural point (MCP) using a neuronavigation system (StealthStation ${ }^{\mathrm{TM}}$, Medtronic). Active contact positions were defined in $\mathrm{X}, \mathrm{Y}, \mathrm{Z}$ space for each hemisphere, and these results were then compared to the clinical outcomes of the individual subjects, who were categorized as responders ( $\geq 50 \%$ seizure reduction) or nonresponders $(<50 \%$ seizure reduction) at 1 -yr postimplant. For individuals who had more than one cathode programmed on a lead ( $\mathrm{n}=81$ left, $\mathrm{n}=79$ right), the active contact location was defined as the interpolation of the 2 active contact positions.

For the new analysis, lead trajectories were assessed qualitatively for proximity to the ANT subregion target, using axial, coronal, sagittal, and lead trajectory plane static images. A novel method was devised to aid in defining the ANT in cases where visualization of anatomic landmarks (ie, AC, fornix, MTT) was difficult. The thalamus was segmented by defining the anterior-posterior (AP) and dorso-ventral (DV) axes at a parasagittal plane through the lead trajectory, and 4 quadrants of an ellipse created by outlining the thalamus using these major axes. When this parasagittal plane is centered on the junction of the MTT with the ventral border of the nucleus, the ANT occupies most of the anterior, superior quadrant and extends to, or just past the AP midpoint of the thalamus (Figure 1).

Lead locations were examined in all subjects for whom adequate postoperative imaging $(\mathrm{n}=103)$ and 1 -yr outcomes $(\mathrm{n}=101)$ were available. Assessments of lead positions were made by 2 raters who were blinded to the outcome data. Each lead trajectory was assigned a score based on the following prospectively defined criteria: $3=$ ideal placement, trajectory passes through the identified subregion target; 2 = slight deviation from ideal placement in one dimension (eg, posterior or medial) but still within a few millimeters of the target region; $1=$ large deviation from ideal placement (either in multiple dimensions or in one dimension); $0=$ trajectory did not appear to traverse the ANT. Representative examples of the range of lead placements (and scores) are shown in Figure 2.

Lead positions were assessed in sagittal, axial, and coronal images (in that hierarchy) and a total score assigned for each subject (range $=0$ 6 for bilateral placement). A score of 4 or greater was considered a good bilateral placement, indicating 2 trajectories near the target region. Scores below 4 were considered "suboptimal" as at least one lead would have been rated as deviating considerably from the "ideal" target. Lead placement scores were then compared to the clinical outcomes during year 1 , defined as the average seizure reduction for 32 -mo reporting periods during the unblinded phase (months 7-12). Because the lead placement scores were ordinal in nature (although they were described numerically) a categorical analysis (Fisher's exact test) was conducted.

\section{RESULTS}

The original targeting analysis revealed that, despite marked variability, average active contact locations were comparable to the coordinates defined as the SANTÉ target $(X=5-6 \mathrm{~mm}$, $\mathrm{Y}=0-2 \mathrm{~mm}$ anterior to $\mathrm{MCP}, \mathrm{Z}=10-12 \mathrm{~mm}$ superior to the intercommissural plane) based on the Schaltenbrand and Wahren atlas. These coordinates represented the target for the center of the 3387 lead (ie, the interspace between contacts 1 and 

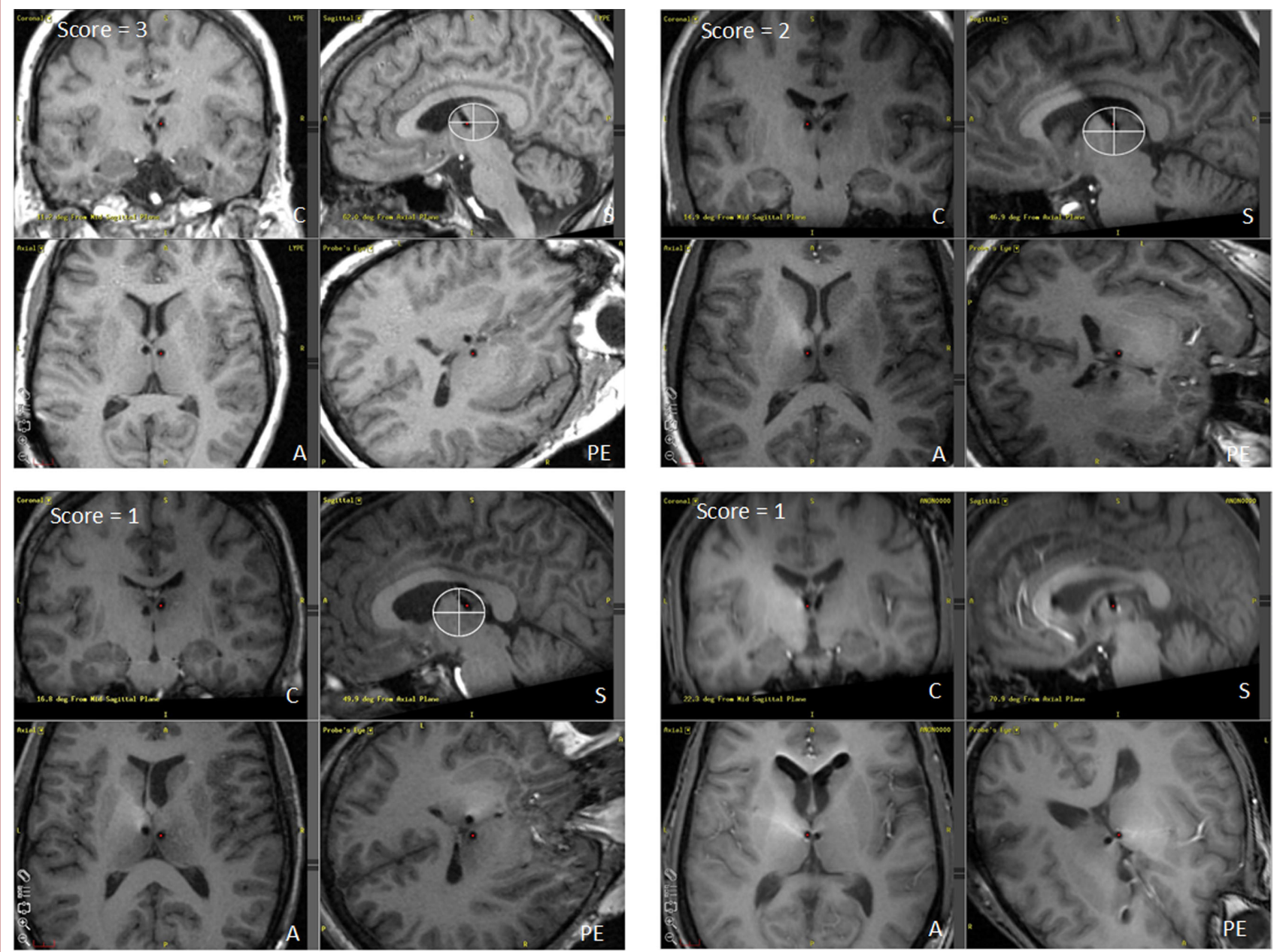

FIGURE 2. Four examples of individual cases from the SANTÉ trial illustrating different lead trajectories and resultant placement scores. Each case includes 4 images: sagittal (S), coronal (C), axial (A), and probe's eye (PE) trajectory views. Representative examples of ideal placement ( $R$ lead) score $=3$ (top left); slight posterior placement $(L$ lead) score $=2$ (top right); very posterior placement ( $R$ lead) score $=1$ (bottom left); and very medial placement $(L$ lead) score $=1$ (bottom right).

2). When compared to the clinical outcomes of the individual subjects, who were categorized as responders or nonresponders at 1 -yr postimplant, there was no apparent relationship between active contact location and categorical outcome, with considerable overlap between the 2 groups (Figure 3).

In the new qualitative analysis of lead trajectory positions, individual scores for bilateral placements ranged from 1 to 6 across the subject cohort. Overall, out of 103 subjects, nearly $70 \%$ had a score of 4 or greater (good bilateral placement), with the most common deviations from the "ideal" target being in the medial and posterior directions (Tables 1 and 2).

The lead placement scores were mapped to each subject's clinical outcome (\% seizure reduction) during year 1 as shown in Figure 4, and assessed against the predefined categories for bilateral placement scores (good vs suboptimal) and clinical response $(>50 \%$ reduction in seizures). These categorical outcome data are summarized in Table 3. Overall, it was found that subjects with a score of 4 or greater (ie, "good" bilateral placement) had a much higher probability of being a clinical responder than those with a score of 3 or less $(43.5 \%$ vs $21.9 \%, P<.05$, Fisher's exact, 2 -sided). When assessed across different subgroups based upon seizure onset location, this trend remained consistent (temporal onset: $50.0 \%$ vs $31.6 \%$; extratemporal onset: $36.4 \%$ vs $7.7 \%$ ) but was not significant due to the small sample sizes.

\section{DISCUSSION}

Accumulating evidence suggests that ANT DBS is an effective, durable therapy for partial onset epilepsy. ${ }^{6}$ The original evaluation of lead placements in the SANTÉ trial, done at the conclusion of the study, did not suggest a relationship between clinical outcomes 

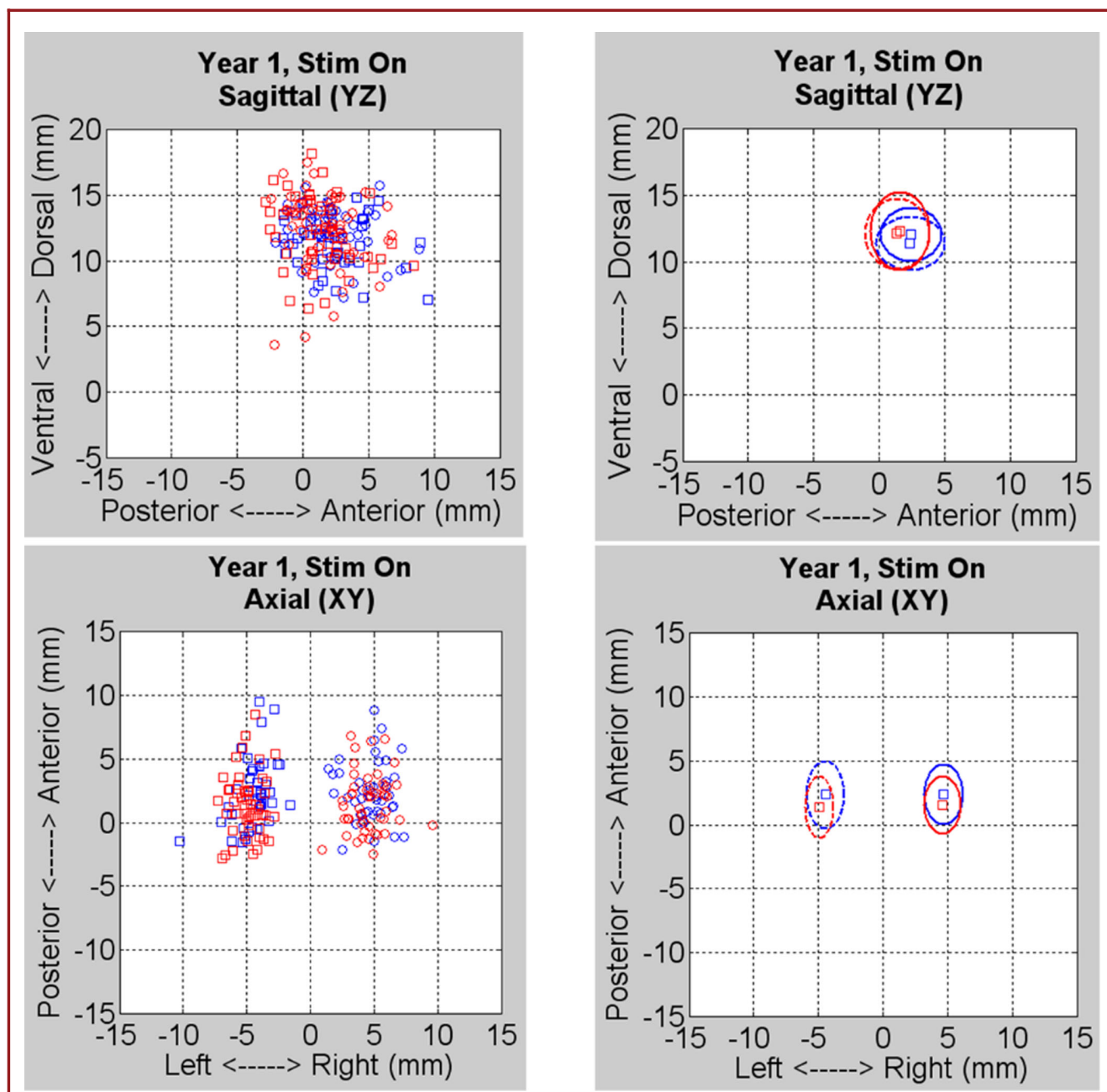

FIGURE 3. Results from the original coordinate-based analysis. Left panels show individual subjects' active contact locations in the sagittal (top) and axial (bottom) planes for right (circles) and left (squares) leads with responders in blue and nonresponders in red ( $n=94:$ responders $=43$, nonresponders $=51$ ). Right panels show median contact positions and one standard deviation from the median for both groups.

\begin{tabular}{|lcc|}
\hline \multicolumn{3}{|l|}{ TABLE 1. Distribution of Bilateral Lead Placement Scores } \\
\hline Bilateral score & Number of subjects & (\%) of subjects \\
\hline 0 & 0 & $0.0 \%$ \\
1 & 2 & $1.9 \%$ \\
2 & 6 & $5.8 \%$ \\
3 & 25 & $24.3 \%$ \\
4 & 24 & $23.3 \%$ \\
5 & 37 & $35.9 \%$ \\
6 & 9 & $8.7 \%$ \\
\hline
\end{tabular}

and active contact location. This analysis was based on contact position in AC-PC stereotactic space and not on location relative to thalamic landmarks. Also, a relatively large number of leads (approximately 80\%) were programmed with multiple cathodes.
TABLE 2. Direction of Individual Lead Placement Deviation From Ideal Target

\begin{tabular}{|lcc|}
\hline Lead placement deviation & Number of leads & (\%) of leads \\
\hline Lateral & 0 & $0.0 \%$ \\
Medial & 95 & $46.1 \%$ \\
Anterior & 5 & $2.4 \%$ \\
Posterior & 53 & $25.7 \%$ \\
\hline
\end{tabular}

To simplify the analysis, the position of the active contact in these cases was defined as the interpolation of the 2 contacts, based upon modeling of the stimulation fields. Given the relatively high stimulation amplitudes (typically 5-7.5 V) and use of multiple contacts on the 3387 lead ( $1.5 \mathrm{~mm}$ contact spacing), the volume 


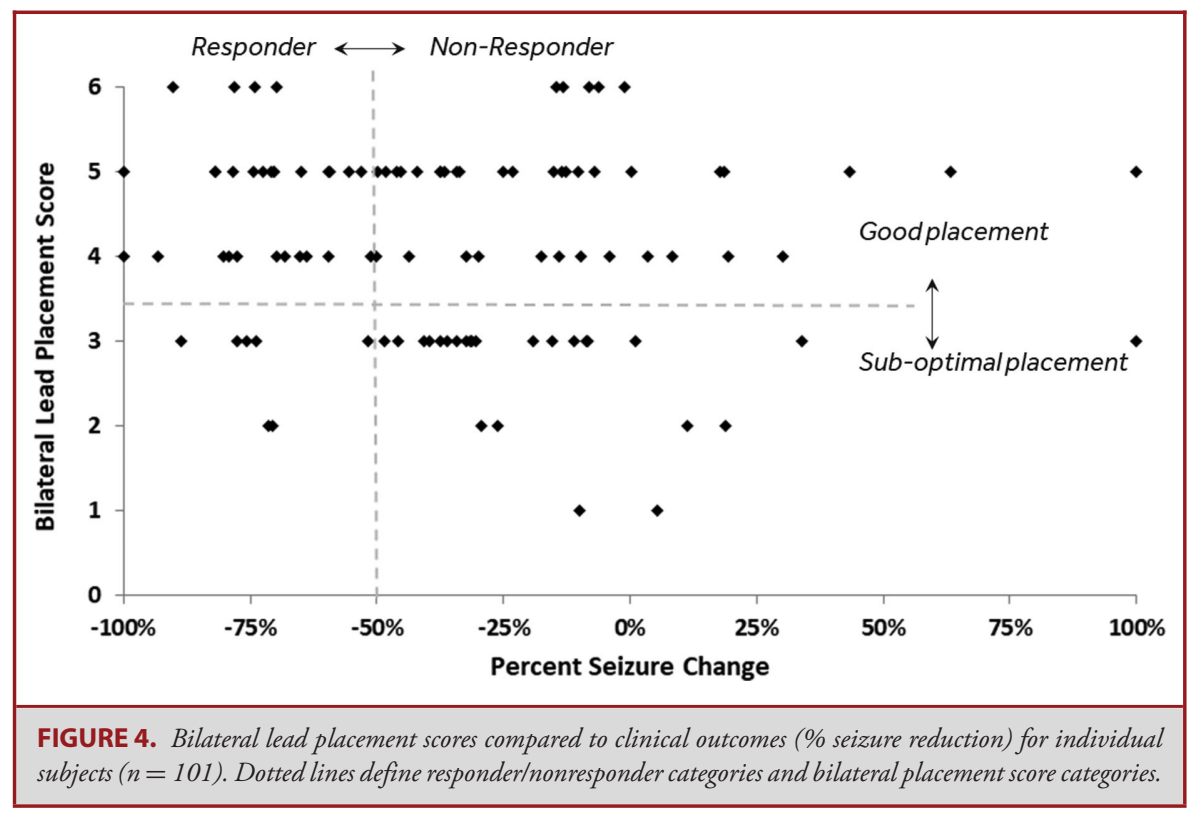

TABLE 3. Distribution of Lead Placement Scores for Responders Versus Nonresponders

\begin{tabular}{|lcc|}
\hline Bilateral score $^{\text {a }}$ & Responder & Nonresponder \\
\hline 3 or $<(n=33)$ & $21.9 \%$ & $78.1 \%$ \\
4 or $>(n=70)$ & $43.5 \%$ & $56.5 \%$ \\
\hline
\end{tabular}

${ }^{a} A$ small group of subjects $(n=6)$ had bilateral scores of 4 , with a score of 3 on one side and 1 on the other. Those subjects were evenly distributed between responders and nonresponders.

of tissue activated (VTA) was likely quite large in some of these subjects. In current practice of ANT DBS, it has become more common to use the Model 3389 lead (0.5 mm spacing) with initial stimulation voltages in the 2 to $3 \mathrm{~V}$ range on a single contact and titrate upward if needed, ${ }^{7}$ based on the size of this nucleus (similar to subthalamic nucleus (STN)) and modeling estimates of the resultant VTA. ${ }^{8}$

\section{Key Results}

In the current analysis, patients who had 2 well-placed leads, based upon their position within the target structure, had a much greater likelihood of being a clinical responder than those who did not, independent of seizure onset location. This result is consistent with experience in other DBS indications, where accurate lead placement at specific locations within the target nucleus is important and associated with better clinical outcomes. ${ }^{9}$ However, in this case, higher placement scores did not always favor being a responder. Therefore, good placement appears to be a necessary, but not sufficient, condition for success. Other, yet unknown, factors also likely influence individual patient outcome.

\section{Interpretation}

This new targeting analysis was motivated by recent studies examining lead location within the ANT, relative to specific landmarks (eg, MTT) and the relationship with clinical outcomes. ${ }^{3,7,10,11}$ Lehtimäki et $\mathrm{al}^{3}$ first identified a subregion in the anterior portion of the nucleus, superior to the MTT, which was associated with greater seizure reduction. The MTT is the main input to the ANT, and the outflow fibers from this structure exit via the anterior thalamic radiations in the lateral portion of the nucleus into the internal capsule. ${ }^{12}$ Stimulation of contacts in this target area therefore likely activates these densely packed axonal populations ${ }^{13,14}$ and, as in other DBS therapies, results in modulation of broader network activity, in this case in the Papez circuit. Based on the prior studies demonstrating a subregion within the ANT may be associated with superior efficacy, we evaluated the SANTÉ dataset in the context of lead trajectory relative to this target area. We a priori defined a rating/grading system that classified the lead trajectories relative to the ANT target region. Trajectories that were through or within a few millimeters of the target region were considered to be good placements given that the stimulation voltages used would likely capture this target region based upon VTA considerations. Trajectories that were more distant from this region were considered suboptimal. Our intent was to develop a methodology useful for evaluating ANT implantation, based on categorical methods, recognizing the variation that has been observed in the ANT DBS target. The fact that our results were in line with the findings from 4 prior, smaller studies was reassuring.

There was likely a learning curve for placement of DBS leads in this specific target during the SANTÉ trial as has been reported for other DBS therapies. ${ }^{15-17}$ Based on recent experience in a multicenter European study (MORE Registry), this DBS 
target appears to have some unique challenges compared to the traditional movement disorders targets in thalamus, subthalamic nucleus, and globus pallidus, due to its location at the base of the ventricle and resultant variability across individual subjects. ${ }^{18}$ For this reason, in the European study 2 approaches to the ANT target were utilized, transventricular and extraventricular, based primarily upon surgeon preference. However, the analysis of lead locations in the first cohort of 73 subjects revealed that only $71 \%$ of the extraventricular lead placements successfully positioned at least one contact into the nucleus, compared to $90 \%$ with the transventricular approach.

All of the implants in the SANTÉ study were done using a transventricular approach, and each case was reviewed by an independent core lab to ensure that at least one contact of each lead was in the target nucleus before subjects could be randomized; $8.2 \%$ of the leads needed to be revised based upon this criterion. However, quadrant or subregion within ANT was not analyzed and replacement was not mandated so long as one contact resided somewhere within the ANT. The clinical results from the SANTÉ trial are comparable to the MORE registry experience using the same transventricular trajectory, and provide support for this approach versus the extraventricular trajectory, even without specifically targeting the area superior to the MTT. However, improved clinical outcomes may be attainable in the future, as a result of our study and those of others ${ }^{3,11,19}$ which appear to be converging on this target region as the most effective stimulation site.

\section{Limitations}

The results presented here have clear limitations due to the retrospective, post hoc nature. However, in the spirit of providing information that can help to improve DBS therapy efficacy and to benefit patients who elect to undergo the surgery and continued follow-up associated with DBS, these results add information to the knowledge base related to this therapy, which at this time remains fairly limited.

\section{Generalizability}

It is universally accepted in movement disorders DBS that accurate placement of leads into the STN, internal globus pallidus, or ventral intermediate nucleus is critical for optimal therapy efficacy. All of these therapies allow for evaluation of lead location during surgery based on test stimulation results. Unfortunately, for the ANT target, there are no reliable acute stimulation induced positive effects, nor side effects, to help assess the position of the leads. However, considering the growing body of evidence related to targeting and clinical outcomes for this therapy, there should be no reason to accept suboptimal lead placements given the ability with current imaging technology to reliably evaluate lead location either intra- or postoperatively. The significant benefit observed during the SANTÉ trial, using widely spaced electrode contacts and suboptimal targeting methods, suggests an opportunity to improve outcomes with more accurate lead placement.

\section{CONCLUSION}

The results from this study suggest that a specific area within the ANT, namely the anterior region, is associated with better clinical outcomes with DBS. Subjects with good bilateral placements in this target had a higher probability of being a clinical responder $(43.5 \%)$ at $1 \mathrm{yr}$ of follow-up, relative to the group with a suboptimal placement $(21.9 \%)$. These findings from the SANTÉ cohort are consistent with earlier reports from smaller studies, and suggest that a certain degree of specificity in lead targeting is critical for ensuring a good clinical outcome.

\section{Funding}

Emory University received a research grant from Medtronic to support this research. Stanford University received a research grant from Medtronic to support this research.

\section{Disclosures}

Dr Gross serves as a consultant to Medtronic, which manufactures the devices used in this project. Dr Gross receives compensation for these services. Unrelated to this work, Dr Gross also received consulting fees from Neuropace, Abbott, Boston Scientific, Sanbio, Zimmer Biomet, Voyager Therapeutics and MRI Interventions and has stock options in Nia Therapeutics. The terms of these arrangements have been reviewed and approved by Emory University in accordance with its conflict of interest policies. Dr Fisher serves as a consultant to Medtronic. Unrelated to the subject of this paper, Dr Fisher owns stock or options in Avails Medical, Cerebral Therapeutics, Eysz, Irody, Smart Monitor and Zeto. Dr Sperling has received research support from Medtronic, SK Life Science, Cavion, Xenon, Takeda, Eisai, Neurelis, Engage Therapeutics, Pfizer, and UCB Pharma; has consulted for Medtronic (fee to institution); CME speaker: WebMD, Medscape, Eisai, Neurology Live and International Medical Press. Dr Giftakis is a clinical employee of Medtronic, the sponsor of the SANTÉ study, and holds stock options. Dr Stypulkowski is a retired employee of Medtronic. He currently serves as a consultant to Medtronic and holds stock options.

\section{REFERENCES}

1. Fisher R, Salanova V, Witt T, et al. Electrical stimulation of the anterior nucleus of thalamus for treatment of refractory epilepsy. Epilepsia. 2010;51(5):899-908.

2. Möttönen T, Katisko J, Haapasalo J, et al. Defining the anterior nucleus of the thalamus (ANT) as a deep brain stimulation target in refractory epilepsy: delineation using 3 T MRI and intraoperative microelectrode recording. Neuroimage Clin. 2015;7:823-829.

3. Lehtimäki K, Möttönen T, Järventausta K, et al. Outcome based definition of the anterior thalamic deep brain stimulation target in refractory epilepsy. Brain Stimul. 2016;9(2):268-275.

4. Wu C, D'Haese PF, Pallavaram S, Dawant BM, Konrad P, Sharan AD. Variations in thalamic anatomy affect targeting in deep brain stimulation for epilepsy. Stereotact Funct Neurosurg. 2016;94(6):387-396.

5. Schaltenbrand G, Wahren W. Atlas for Stereotaxy of the Human Brain. Stuttgart: Georg Thieme Verlag; 1977.

6. Salanova V, Witt T, Worth R, et al. Long-term efficacy and safety of thalamic stimulation for drug-resistant partial epilepsy. Neurology. 2015;84(10):1017-1025.

7. Guo W, Koo BB, Kim JH, et al. Defining the optimal target for anterior thalamic deep brain stimulation in patients with drug-refractory epilepsy [published online ahead of print: May 8, 2020]. J Neurosurg. doi: 10.3171/2020.2.JNS193226.

8. Butson CR, Cooper SE, Henderson JM, Wolgamuth B, McIntyre CC. Probabilistic analysis of activation volumes generated during deep brain stimulation. Neuroimage. 2011;54(3):2096-2104.

9. Welter ML, Schüpbach M, Czernecki V, et al. Optimal target localization for subthalamic stimulation in patients with Parkinson disease. Neurology. 2014;82(15):1352-1361. 
10. Koeppen JA, Nahravani F, Kramer M, et al. Electrical stimulation of the anterior thalamus for epilepsy: clinical outcome and analysis of efficient target. Neuromodulation. 2019;22(4):465-471.

11. Schaper F, Plantinga BR, Colon AJ, et al. Deep brain stimulation in epilepsy: a role for modulation of the mammillothalamic tract in seizure control? Neurosurgery. 2020;87(3):602-610

12. Xiao D, Barbas H. Pathways for emotions and memory I. Input and output zones linking the anterior thalamic nuclei with prefrontal cortices in the rhesus monkey. Thalamus Relat Syst. 2002;2(1):21-32.

13. Montgomery EB, Jr, Gale JT. Mechanisms of action of deep brain stimulation (DBS). Neurosci Biobehav Rev. 2008;32(3):388-407.

14. Johnson LA, Vitek JL. Deep brain stimulation: mechanisms of action. In: Winn RH ed. Youmans and Winn Neurological Surgery. New York, NY: Elsevier. Vol. 91, 6th ed, 2011:635-646.

15. Simuni T, Jaggi JL, Mulholland H, et al. Bilateral stimulation of the subthalamic nucleus in patients with Parkinson disease: a study of efficacy and safety. J Neurosurg. 2002;96(4):666-672.

16. Starr PA, Christine CW, Theodosopoulos PV, et al. Implantation of deep brain stimulators into the subthalamic nucleus: technical approach and magnetic resonance imaging-verified lead locations. J Neurosurg. 2002;97(2): 370-387.

17. Greenberg BD, Gabriels LA, Malone DA Jr, et al. Deep brain stimulation of the ventral internal capsule/ventral striatum for obsessive-compulsive disorder: worldwide experience. Mol Psychiatry. 2010;15(1):64-79.

18. Lehtimäki K, Coenen VA, Gonçalves Ferreira A, et al. The surgical approach to the anterior nucleus of thalamus in patients with refractory epilepsy: experience from the international multicenter registry (MORE). Neurosurgery. 2019;84(1):141150 .

19. Krishna V, King NK, Sammartino F, et al. Anterior nucleus deep brain stimulation for refractory epilepsy: insights into patterns of seizure control and efficacious target. Neurosurgery. 2016;78(6):802-811.

Neurosurgery Speaks! Audio abstracts available for this article at www. neurosurgery-online.com.

\section{COMMENTS}

$1 \mathrm{~s}$ with other DBS targets, it is logical that there may be a variation $\Delta$ in response based on lead location, and it is intriguing that direct visualization of structural and anatomic features seemed to correlate better with outcome than traditional AC-PC targeting. However, while statistically significant, the post-hoc nature of the study and relatively small differences observed make it difficult to interpret how significant the effect might be. In addition, the definition of "ideal placement" relative to MTT is highly subjective (including descriptive judgement calls for "slight" vs. "large" displacement) and the definition of "good" placement as a score of 4 (which combines "slight" displacement on both sides with "large" displacement on one side) is somewhat arbitrary. If would have been much more compelling if the authors had used a more quantitative measure, such as identifying the putative target location in each subject and measuring distance of the active contacts from it. It is also worth noting that a majority of subjects in both groups were nonresponders, and a number of subjects with "good" bilateral placement actually got worse. The data imply that targeting may indeed impact efficacy, but more evidence will be necessary to support the efficacy of this particular target.

Jonathan P. Miller Cleveland, Ohio, USA

The authors present a thorough analysis of DBS electrode location within the anterior thalamic nucleus in $>100$ epilepsy patients who were monitored for degree of symptomatic improvement for more than a year after the implant. Not surprisingly, precise targeting was associated with remarkably higher chance of meaningful clinical response with almost 2-fold difference between those with optimal and suboptimal location of stimulating contacts.

To further improve individual outcomes, one will need to develop either an easily identifiable set of radiographic landmarks, or a reliable neurophysiological indicator of the target. Alternatively, one may use technological advancement in electrode leads (directional, segmented, etc) to compensate for inaccurate targeting.

It would be interesting to extend this analysis to hundreds of patients implanted with ANT DBS outside of SANTÉ trial to see if the authors' findings are indeed valid.

Konstantin Slavin Chicago, Illinois, USA 